\title{
Intrafamilial variability and clinical heterogeneity in a family with PLA2G6-associated neurodegeneration
}

\author{
Jong Kyu Park ${ }^{1}$, Jinyoung Youn ${ }^{2,3}$, Jin Whan Cho ${ }^{2,3}$ \\ ${ }^{1}$ Department of Neurology, Soonchunhyang University Cheonan Hospital, Soonchunhyang University College of Medicine, \\ Cheonan, Korea \\ ${ }^{2}$ Department of Neurology, Samsung Medical Center, Sungkyunkwan University School of Medicine, Seoul, Korea \\ ${ }^{3}$ Neuroscience Center, Samsung Medical Center, Seoul, Korea
}

Received: August 8, 2019

Revised: August 21, 2019

Accepted: August 23, 2019

Corresponding author:

Jin Whan Cho

Department of Neurology,

Samsung Medical Center,

Sungkyunkwan University

School of Medicine, 81 Irwon-

ro, Gangnam-gu, Seoul 06351,

Korea

Tel: +82-2-3410-3599

E-mail:

jinwhan.cho@samsung.com

\begin{abstract}
Phospholipase A2 group VI (PLA2G6)-associated neurodegeneration (PLAN) is an autosomal recessive neurodegenerative disease with a wide clinical spectrum; however, the genotype-phenotype correlation is unknown. Here, we report different phenotypes in one family with the same genotype. A 28-year-old male presented with slowly progressive gait disturbance with spasticity. Onset occurred at 11 years. Interestingly, his younger brother, a 24-year-old male, presented with progressive Parkinsonism, which began at 22 years. He showed excellent response to levodopa but developed a fluctuating medication response and levodopa-induced dyskinesia 1 year after starting levodopa medication. He also demonstrated hyperreflexia, but no spasticity. Dopamine transporter imaging showed reduced uptake in the bilateral putamen. In whole-exome sequencing and Sanger sequencing, a homozygous pathogenic variant (p. R747W) in the PLA2G6 gene was detected in both cases. Despite different clinical features, both subjects had hyperreflexia during the examination and claval hypertrophy was shown on the brain magnetic resonance imaging.
\end{abstract}

Keywords: PLA2G6; Parkinson disease; Genetic analysis

\section{INTRODUCTION}

Phospholipase A2 group VI (PLA2G6)-associated neurodegeneration (PLAN) is an autosomal recessive neurodegenerative disease with multiple phenotypes, including infantile neuroaxonal dystrophy, neurodegeneration with brain iron accumulation, and dystonia-Parkinsonism $[1,2]$. The clinical spectrum of PLAN has been proposed to include various phenotypes associ-

This is an Open Access article distributed under the terms of the Creative Commons Attribution Non-Commercial License (http:// creativecommons.org/licenses/ by-nc/4.0/). ated with PLA2G6 mutations. Although various phenotypes are known in patients with PLAN, genotype-phenotype correlation has yet to be clearly elucidated. Further, there are no reports demonstrating various phenotypes even in one family with the same genotype. Here, we describe a family with the same genotype of PLAN, but with phenotypic variability. 


\section{CASE REPORTS}

\section{Subject 1}

A 28-year-old male with progressive spastic gait disturbance that began at the age of 11 presented at Samsung Medical Center, Seoul, Korea. He was born to healthy consanguine-

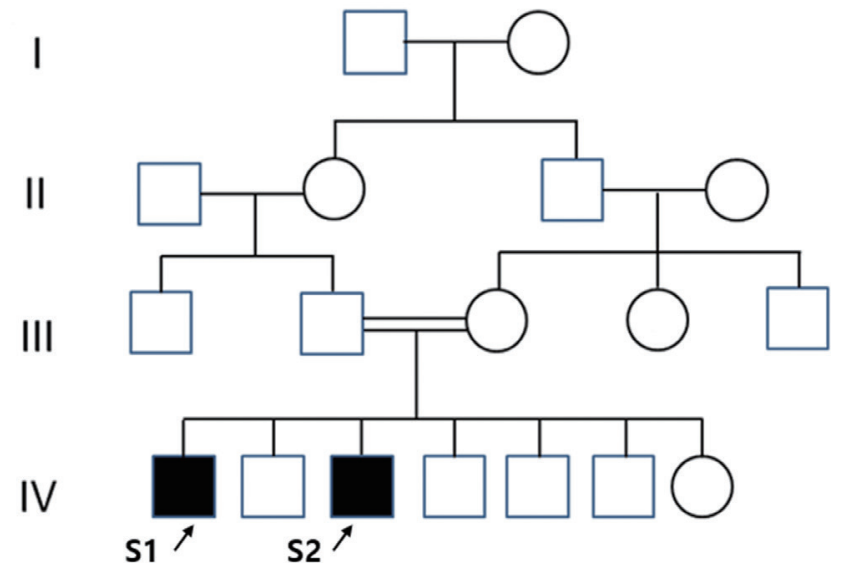

Fig. 1. Pedigree of the index subjects: black symbols indicate affected individuals and arrows indicate subjects. ous parents of Arabian ancestry. He had no history of preterm delivery or perinatal injury, and his developmental milestones were normal. There was no family history of movement disorders, except in his younger brother (Subject 2). The familial pedigree is illustrated in Fig. 1. He was normal in cognitive and language skills and finished his master's degree in college. The only accompanying symptom was urinary urgency and incontinence.

When he came to our clinic, spasticity was present in the hip adductor muscles, as well as in the knee and ankle joints. Sensory disturbance of lower limbs was absent. Brisk reflexes were present on both limbs, and pathologic reflexes, such as Hoffman sign, ankle clonus and Babinski sign, were also present. Grade 4 mild muscle weakness was present with both ankle dorsiflexions. He demonstrated spastic gait with dystonic ankle movements interfering speed of gait, but no rigidity or bradykinesia. A nerve conduction study on lower limbs was normal. There was no iron accumulation, but claval hypertrophy was shown on the brain magnetic resonance imaging (MRI) (Fig. 2A). The cervical through thoracic spinal MRI was normal.
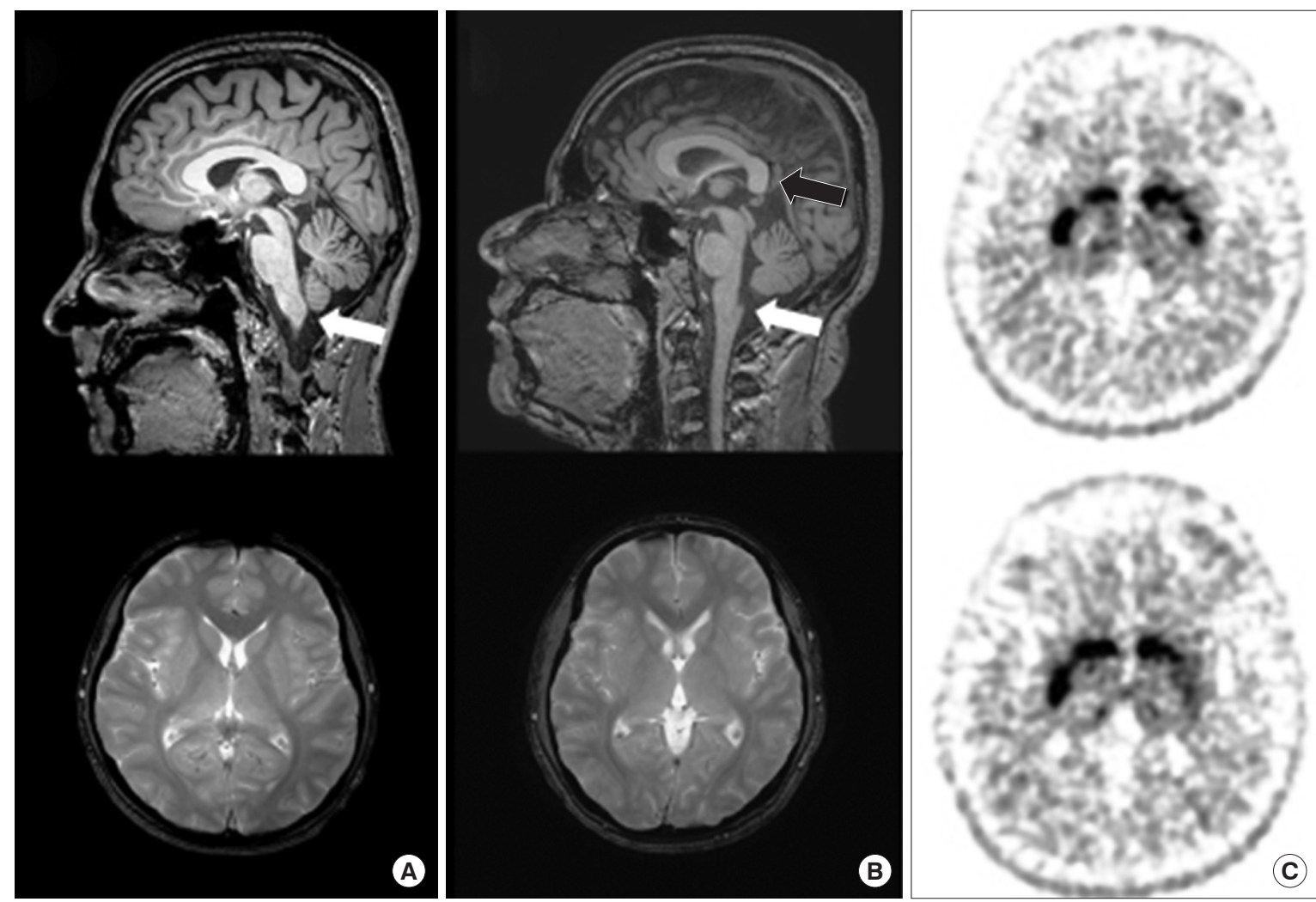

Fig. 2. (A) T2 fluid attenuated inversion recovery (FLAIR) midsagittal section image of subject 1 shows claval hypertrophy (white arrow). (B) T2 FLAIR midsagittal section image of subject 2 shows claval hypertrophy (white arrow) and a vertical corpus callosum (black arrow). (C) Dopamine transporter image of subject 2 demonstrated significantly reduced uptake in the bilateral putamen. 


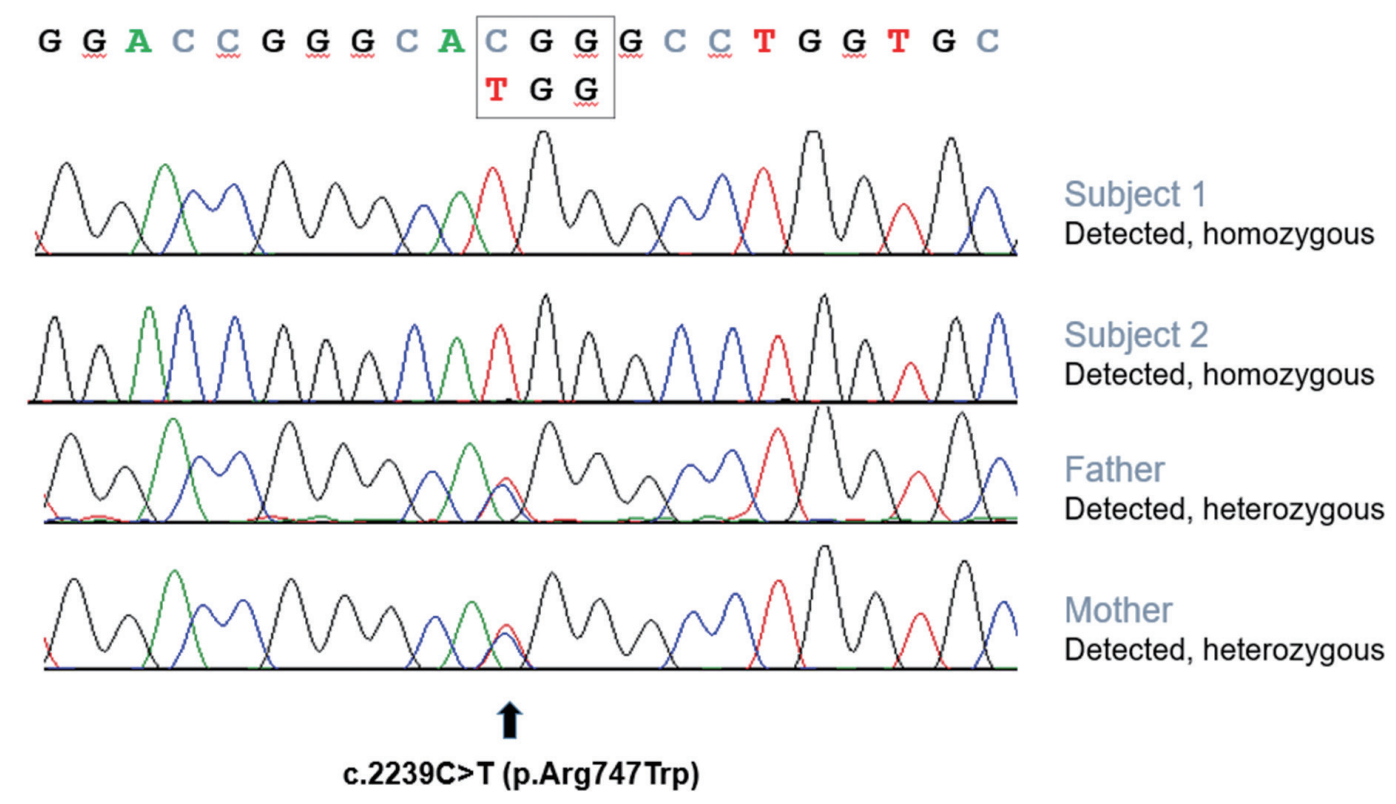

Fig. 3. Sequencing analysis of index subjects showing homozygous variants in phospholipase A2 group VI (PLA2G6). Analysis of parental samples demonstrated that the PLA2G6 variants were heterozygous, which is consistent with autosomal recessive inheritance.

Whole exome sequencing for subject 1 revealed a homozygous c.2239C > T (p.R747W) pathogenic variant in the PLA2G6 gene. Sanger sequencing showed that both of the parents were heterozygous for this variant (Fig. 3). His gait disturbance did not respond with levodopa treatment but partially improved with baclofen ( $30 \mathrm{mg} /$ day) and botulinum toxin type $A$ injection on the tibialis posterior muscle (50 units, each).

\section{Subject 2}

The younger brother of subject 1, a 24-year-old male, presented at our clinic with slowly progressive Parkinsonism that began at the age of 22. Like subject 1 , he had no history of preterm delivery or perinatal injury, and the developmental milestones were normal. Urinary urgency was combined, while general cognition was normal.

On the neurologic exam, bradykinesia and rigidity were present in both limbs, but more prominently on the left side. Additionally, rest tremor was seen in both arms. Deep tendon reflexes were brisk and the Babinski sign was present in both lower limbs. F-18 fluorinated-N-3-fluoropropyl-2-b-carboxymethoxy-3-b-(4-iodophenyl)nortropane positron emission tomography (FP-CIT PET) demonstrated significantly reduced uptake in the bilateral putamen (Fig. 2C). Additionally, corpus callosum changes and claval hypertrophy were seen on the brain MRI (Fig. 2B).

We performed Sanger sequencing of the PLA2G6 gene, and the same homozygous pathogenic variant (p.R747W) was detected (Fig. 3). His Parkinsonism improved dramatically with levodopa medication, but he developed wearing-off phenomenon and levodopa-induced dyskinesia within 1 year from the levodopa start date.

\section{DISCUSSION}

Here, we described two different phenotypes of the same genotype of PLAN in one family. One phenotype was levodopa-responsive Parkinsonism and the other was spastic paraplegia. Both shared hyperreflexia on lower limbs and pyramidal tract syndrome, but totally different main phenotypic presentations in the same genotype are rarely reported. Interestingly, PLAN covers various phenotypes of PLA2G6 mutation, and various phenotypes of PLAN are described according to the age of onset (e.g., infantile neuroaxonal dystrophy occurs in childhood and dystonia-Parkinsonism occurs in early adulthood). However, even though various phenotypes of PLAN have been reported, there are currently insufficient studies on phenotype-genotype correlation. Further, our patients were similar in age but presented with completely different phenotypes in a single family with the same genotype, unlike various phenotypes in PLAN. To the best of our knowledge, this is the first report of intrafamilial variability in monogenic PLAN. The mechanisms responsible for determination of phenotypes are not yet known. 
The first phenotype of our subjects was levodopa-responsive Parkinsonism. Parkinsonism is a commonly reported symptom in PLAN patients, and the same genotype ( $p$. R747W) has been previously reported in a patient with levodopa-responsive Parkinsonism [3,4]. In accordance with previously reported PLAN cases with Parkinsonism, our case also showed normal birth, achieved normal milestones, and symptoms began in adulthood. All cases progressed rapidly after the onset and showed a dramatic response to levodopa therapy, but an impulse control disorder or early dyskinesia appeared. Additionally, our case also revealed presynaptic dopaminergic loss by FP-CIT PET imaging study.

In terms of spastic paraplegia, 60 hereditary spastic paraplegia (HSP)-related genes have been reported, but the underlying causes of HSP still remain unidentified [5]. Recently, PLAN with phenotype of complicated HSP was reported [6,7], but pure form spastic paraplegia was not reported. With whole exome sequencing, we excluded associated genes for HSP in subject 1; thus, we suggest pure form spastic paraplegia is a new phenotype of PLAN based on our case.

Despite different main phenotypes of our patients, both subjects also demonstrated common clinical features. First, pyramidal tract syndrome was seen in both subjects. Additionally, both cases had claval hypertrophy (Fig. 2A, B). In terms of brain imaging, abnormal iron accumulation is considered as a characteristic sign of neurodegeneration with brain iron accumulation, but iron accumulation is no longer a pathognomonic finding in PLA2G6 mutations. Similarly, our cases did not show iron accumulation in brain. Additionally, various MRI findings, including cerebral and/or cerebellar atrophy, corpus callosum changes, or claval hypertrophy, could be observed in patients with PLAN [8]. Subject 2 showed corpus callosum changes as well as claval hypertrophy (Fig. 2B). Therefore, pyramidal tract syndrome and claval hypertrophy could be the diagnostic clue for PLAN to overcome the phenotypic diversity.

In conclusion, the affected patients of PLA2G6 mutation showed clinical heterogeneity within a family. PLA2G6 mutation can present as a pure form of spastic paraplegia or L-dopa responsive Parkinsonism with motor fluctuation. Additionally, we describe a previously unrecognized phenotype of PLA2G6 mutations that is pure form spastic paraplegia.

\section{CONFLICTS OF INTEREST}

No potential conflict of interest relevant to this article was reported.

\section{ORCID}

Jong Kyu Park https://orcid.org/0000-0002-9261-790X

Jinyoung Youn https://orcid.org/0000-0003-3350-5032

Jin Whan Cho https://orcid.org/0000-0002-9145-6729

\section{REFERENCES}

1. Guo YP, Tang BS, Guo JF. PLA2G6-associated neurodegeneration (PLAN): review of clinical phenotypes and genotypes. Front Neurol 2018;9:1100.

2. Lee JH, Park J, Ryu HS, Park H, Kim YE, Hong JY, et al. Clinical heterogeneity of atypical pantothenate kinase-associated neurodegeneration in Koreans. J Mov Disord 2016; 9:20-7.

3. Paisan-Ruiz C, Li A, Schneider SA, Holton JL, Johnson R, Kidd $D$, et al. Widespread Lewy body and tau accumulation in childhood and adult onset dystonia-parkinsonism cases with PLA2G6 mutations. Neurobiol Aging 2012;33:814-23.

4. Giri A, Guven G, Hanagasi H, Hauser AK, Erginul-Unaltuna $\mathrm{N}$, Bilgic $\mathrm{B}$, et al. PLA2G6 mutations related to distinct phenotypes: a new case with early-onset parkinsonism. Tremor Other Hyperkinet Mov (N Y) 2016;6:363.

5. de Souza PVS, de Rezende Pinto WBV, de Rezende Batistella GN, Bortholin T, Oliveira ASB. Hereditary spastic paraplegia: clinical and genetic hallmarks. Cerebellum 2017;16:525-51.

6. Ozes B, Karagoz N, Schule R, Rebelo A, Sobrido MJ, Harmuth F, et al. PLA2G6 mutations associated with a continuous clinical spectrum from neuroaxonal dystrophy to hereditary spastic paraplegia. Clin Genet 2017;92:534-9.

7. Koh K, Ichinose Y, Ishiura H, Nan H, Mitsui J, Takahashi J, et al. PLA2G6-associated neurodegeneration presenting as a complicated form of hereditary spastic paraplegia. J Hum Genet 2019;64:55-9.

8. Karkheiran S, Shahidi GA, Walker RH, Paisan-Ruiz C. PLA2G6-associated dystonia-parkinsonism: case report and literature review. Tremor Other Hyperkinet Mov (N Y) 2015;5:317. 